\title{
A RESULT CONCERNING DERIVATIONS IN PRIME RINGS
}

\author{
MAJA Fošner AND NINA PERŠIN \\ University of Maribor, Slovenia
}

\begin{abstract}
A classical result of Herstein asserts that any Jordan derivation on a prime ring of characteristic different from two is a derivation. It is our aim in this paper to prove the following result, which is in the spirit of Herstein's theorem. Let $R$ be a prime ring with $\operatorname{char}(R)=0$ or $4<\operatorname{char}(R)$, and let $D: R \rightarrow R$ be an additive mapping satisfying either the relation $D\left(x^{3}\right)=D\left(x^{2}\right) x+x^{2} D(x)$ or the relation $D\left(x^{3}\right)=D(x) x^{2}+x D\left(x^{2}\right)$ for all $x \in R$. In both cases $D$ is a derivation.
\end{abstract}

This research has been motivated by the recent work of Vukman ([14]). Throughout, $R$ will represent an associative ring with center $Z(R)$. As usual we write $[x, y]$ for $x y-y x$. Given an integer $n \geq 2$, a ring $R$ is said to be $n$ torsion free, if for $x \in R, n x=0$ implies $x=0$. Recall that a ring $R$ is prime if for $a, b \in R, a R b=(0)$ implies that either $a=0$ or $b=0$ and is semiprime in case $a R a=(0)$ implies $a=0$. An additive mapping $D: R \rightarrow R$, where $R$ is an arbitrary ring, is called a derivation if $D(x y)=D(x) y+x D(y)$ holds for all pairs $x, y \in R$ and is called a Jordan derivation in case $D\left(x^{2}\right)=D(x) x+x D(x)$ is fulfilled for all $x \in R$. A derivation $D$ is inner in case there exists $a \in R$, such that $D(x)=[x, a]$ holds for all $x \in R$. Every derivation is a Jordan derivation. The converse is in general not true. A classical result of Herstein ([11]) asserts that any Jordan derivation on a 2-torsion free prime ring is a derivation. A brief proof of Herstein's result can be found in [7]. Cusack ([10]) generalized Herstein's result to 2-torsion free semiprime rings (see also [3] for an alternative proof). Let us point out that Beidar, Brešar, Chebotar and Martindale ([1]) have considerably generalized Herstein's theorem. A generalization of Herstein's theorem can be found also in [8].

2010 Mathematics Subject Classification. 16W10, 46K15, $39 \mathrm{~B} 05$.

Key words and phrases. Prime ring, semiprime ring, derivation, Jordan derivation, Jordan triple derivation, functional identity. 
Let us start with the following result proved by Brešar ([4]).

TheOREM 1. Let $R$ be a 2-torsion free semiprime ring and let $D: R \rightarrow R$ be an additive mapping satisfying the relation

$$
D(x y x)=D(x) y x+x D(y) x+x y D(x)
$$

for all pairs $x, y \in R$. In this case $D$ is a derivation.

An additive mapping $D: R \rightarrow R$, where $R$ is an arbitrary ring, satisfying the relation (1) for all pairs $x, y \in R$ is called a Jordan triple derivation. One can easily prove that any Jordan derivation $D$ on an arbitrary 2-torsion free ring is a Jordan triple derivation (see, for example, [7]), which means that Theorem 1 generalizes Cusack's generalization of Herstein's theorem. Theorem 1 has been recently generalized in [9]. Let us point out that Brešar's result, we have just mentioned above, has motivated many results (see [15]).

Motivated by Theorem 1 Vukman ([14]) has recently proved the following theorem.

TheOREM 2. Let $R$ be a 2-torsion free semiprime ring and let $D: R \rightarrow R$ be an additive mapping. Suppose that either

$$
D(x y x)=D(x y) x+x y D(x)
$$

or

$$
D(x y x)=D(x) y x+x D(y x)
$$

holds for all pairs $x, y \in R$. In both cases $D$ is a derivation.

Putting $y=x$ in (1), (2) and (3) we obtain

$$
\begin{gathered}
D\left(x^{3}\right)=D(x) x^{2}+x D(x) x+x^{2} D(x), \\
D\left(x^{3}\right)=D\left(x^{2}\right) x+x^{2} D(x)
\end{gathered}
$$

and

$$
D\left(x^{3}\right)=D(x) x^{2}+x D\left(x^{2}\right) .
$$

The relation (4) has been considered in [13] and [1] (actually in [1] much more general situation has been studied). It is our aim in this paper to consider the last two relations.

THEOREM 3. Let $R$ be a prime ring with $\operatorname{char}(R)=0$ or $4<\operatorname{char}(R)$ and let $D: R \rightarrow R$ be an additive mapping satisfying either the relation

$$
D\left(x^{3}\right)=D\left(x^{2}\right) x+x^{2} D(x)
$$

or the relation

$$
D\left(x^{3}\right)=D(x) x^{2}+x D\left(x^{2}\right) .
$$

for all $x \in R$. In both cases $D$ is a derivation. 
Theorem 3 is obtained as an application of the theory of functional identities (Brešar-Beidar-Chebotar theory). In particular, we shall use some ideas from the paper of Beidar and Fong [2] where bijective additive mappings preserving a fixed polynomial are characterized. The theory of functional identities considers set-theoretic mappings on rings that satisfy some identical relations. When treating such relations one usually concludes that the form of the mappings involved can be described, unless the ring is very special. We refer the reader to [5] for an introductory account on functional identities and to [6] for full treatment of this theory. For the proof of Theorem 3 we need Theorem 4 which might be of independent interest.

Let $R$ be an algebra over a commutative ring $\phi$ and let

$$
p\left(x_{1}, x_{2}, x_{3}\right)=\sum_{\pi \in S_{3}} x_{\pi(1)} x_{\pi(2)} x_{\pi(3)}
$$

be a fixed multilinear polynomial in noncommuting indeterminates $x_{i}$ over $\phi$. Here $S_{3}$ stands for the symmetric group of order 3 . Let $\mathcal{L}$ be a subset of $R$ closed under $p$, i.e., $p\left(\bar{x}_{3}\right) \in \mathcal{L}$ for all $x_{1}, x_{2}, x_{3} \in \mathcal{L}$, where $\bar{x}_{3}=\left(x_{1}, x_{2}, x_{3}\right)$. We shall consider a mapping $D: \mathcal{L} \rightarrow R$ satisfying

$$
D\left(p\left(\bar{x}_{3}\right)\right)=\sum_{\pi \in S_{3}} D\left(x_{\pi(1)} x_{\pi(2)}\right) x_{\pi(3)}+\sum_{\pi \in S_{3}} x_{\pi(1)} x_{\pi(2)} D\left(x_{\pi(3)}\right)
$$

for all $x_{1}, x_{2}, x_{3} \in \mathcal{L}$. Let us mention that the idea of considering the expression $\left[p\left(\bar{x}_{3}\right), p\left(\bar{y}_{3}\right)\right]$ in its proof is taken from [2].

TheOREM 4. Let $\mathcal{L}$ be a 6 -free Lie subring of $R$ closed under $p$. If $D$ : $\mathcal{L} \rightarrow R$ is an additive mapping satisfying (8), then $D$ is a derivation.

Proof. For any $a \in R$ and $\bar{x}_{3} \in \mathcal{L}^{3}$ we have

$$
\left[p\left(\bar{x}_{3}\right), a\right]=p\left(\left[x_{1}, a\right], x_{2}, x_{3}\right)+p\left(x_{1},\left[x_{2}, a\right], x_{3}\right)+p\left(x_{1}, x_{2},\left[x_{3}, a\right]\right),
$$

and therefore

$$
\begin{aligned}
D\left[p\left(\bar{x}_{3}\right), a\right]= & \sum_{\pi \in S_{3}} D\left[x_{\pi(1)} x_{\pi(2)}, a\right] x_{\pi(3)}+\sum_{\pi \in S_{3}} D\left(x_{\pi(1)} x_{\pi(2)}\right)\left[x_{\pi(3)}, a\right] \\
& +\sum_{\pi \in S_{3}}\left[x_{\pi(1)} x_{\pi(2)}, a\right] D\left(x_{\pi(3)}\right)+\sum_{\pi \in S_{3}} x_{\pi(1)} x_{\pi(2)} D\left[x_{\pi(3)}, a\right] .
\end{aligned}
$$

In particular

$$
\begin{aligned}
D & {\left[p\left(\bar{x}_{3}\right), p\left(\bar{y}_{3}\right)\right] } \\
= & \sum_{\pi \in S_{3}} D\left[x_{\pi(1)} x_{\pi(2)}, p\left(\bar{y}_{3}\right)\right] x_{\pi(3)}+\sum_{\pi \in S_{3}} D\left(x_{\pi(1)} x_{\pi(2)}\right)\left[x_{\pi(3)}, p\left(\bar{y}_{3}\right)\right] \\
& +\sum_{\pi \in S_{3}}\left[x_{\pi(1)} x_{\pi(2)}, p\left(\bar{y}_{3}\right)\right] D\left(x_{\pi(3)}\right)+\sum_{\pi \in S_{3}} x_{\pi(1)} x_{\pi(2)} D\left[x_{\pi(3)}, p\left(\bar{y}_{3}\right)\right] .
\end{aligned}
$$


Using

$$
\begin{aligned}
& D\left[x_{\pi(1)} x_{\pi(2)}, p\left(\bar{y}_{3}\right)\right]=-D\left[p\left(\bar{y}_{3}\right), x_{\pi(1)} x_{\pi(2)}\right] \\
& =\sum_{\sigma \in S_{3}} D\left[x_{\pi(1)} x_{\pi(2)}, y_{\sigma(1)} y_{\sigma(2)}\right] y_{\sigma(3)}+\sum_{\sigma \in S_{3}} D\left(y_{\sigma(1)} y_{\sigma(2)}\right)\left[x_{\pi(1)} x_{\pi(2)}, y_{\sigma(3)}\right] \\
& \quad+\sum_{\sigma \in S_{3}}\left[x_{\pi(1)} x_{\pi(2)}, y_{\sigma(1)} y_{\sigma(2)}\right] D\left(y_{\sigma(3)}\right)+\sum_{\sigma \in S_{3}} y_{\sigma(1)} y_{\sigma(2)} D\left[x_{\pi(1)} x_{\pi(2)}, y_{\sigma(3)}\right]
\end{aligned}
$$

and

$$
\begin{aligned}
& D\left[x_{\pi(3)}, p\left(\bar{y}_{3}\right)\right]=-D\left[p\left(\bar{y}_{3}\right), x_{\pi(3)}\right] \\
& =\sum_{\sigma \in S_{3}} D\left[x_{\pi(3)}, y_{\sigma(1)} y_{\sigma(2)}\right] y_{\sigma(3)}+\sum_{\sigma \in S_{3}} D\left(y_{\sigma(1)} y_{\sigma(2)}\right)\left[x_{\pi(3)}, y_{\sigma(3)}\right] \\
& \quad+\sum_{\sigma \in S_{3}}\left[x_{\pi(3)}, y_{\sigma(1)} y_{\sigma(2)}\right] D\left(y_{\sigma(3)}+\sum_{\sigma \in S_{3}} y_{\sigma(1)} y_{\sigma(2)} D\left[x_{\pi(3)}, y_{\sigma(3)}\right]\right.
\end{aligned}
$$

in (9) we have

$$
\begin{aligned}
D\left[p\left(\bar{x}_{3}\right), p\left(\bar{y}_{3}\right)\right]= & \sum_{\pi \in S_{3}} \sum_{\sigma \in S_{3}} D\left[x_{\pi(1)} x_{\pi(2)}, y_{\sigma(1)} y_{\sigma(2)}\right] y_{\sigma(3)} x_{\pi(3)} \\
& +\sum_{\pi \in S_{3}} \sum_{\sigma \in S_{3}} D\left(y_{\sigma(1)} y_{\sigma(2)}\right)\left[x_{\pi(1)} x_{\pi(2)}, y_{\sigma(3)}\right] x_{\pi(3)} \\
& +\sum_{\pi \in S_{3}} \sum_{\sigma \in S_{3}}\left[x_{\pi(1)} x_{\pi(2)}, y_{\sigma(1)} y_{\sigma(2)}\right] D\left(y_{\sigma(3)}\right) x_{\pi(3)} \\
& +\sum_{\pi \in S_{3}} \sum_{\sigma \in S_{3}} y_{\sigma(1)} y_{\sigma(2)} D\left[x_{\pi(1)} x_{\pi(2)}, y_{\sigma(3)}\right] x_{\pi(3)} \\
& +\sum_{\pi \in S_{3}} \sum_{\sigma \in S_{3}} D\left(x_{\pi(1)} x_{\pi(2)}\right)\left[x_{\pi(3)}, y_{\sigma(1)} y_{\sigma(2)} y_{\sigma(3)}\right] \\
& +\sum_{\pi \in S_{3}} \sum_{\sigma \in S_{3}}\left[x_{\pi(1)} x_{\pi(2)}, y_{\sigma(1)} y_{\sigma(2)} y_{\sigma(3)}\right] D\left(x_{\pi(3)}\right) \\
& +\sum_{\pi \in S_{3}} \sum_{\sigma \in S_{3}} x_{\pi(1)} x_{\pi(2)} D\left[x_{\pi(3)}, y_{\sigma(1)} y_{\sigma(2)}\right] y_{\sigma(3)} \\
& +\sum_{\pi \in S_{3}} \sum_{\sigma \in S_{3}} x_{\pi(1)} x_{\pi(2)} D\left(y_{\sigma(1)} y_{\sigma(2)}\right)\left[x_{\pi(3)}, y_{\sigma(3)}\right] \\
& +\sum_{\pi \in S_{3}} \sum_{\sigma \in S_{3}} x_{\pi(1)} x_{\pi(2)}\left[x_{\pi(3)}, y_{\sigma(1)} y_{\sigma(2)}\right] D\left(y_{\sigma(3)}\right) \\
& +\sum_{\pi \in S_{3}} \sum_{\sigma \in S_{3}} x_{\pi(1)} x_{\pi(2)} y_{\sigma(1)} y_{\sigma(2)} D\left[x_{\pi(3)}, y_{\sigma(3)}\right]
\end{aligned}
$$


If we replace the roles of $\pi$ and $\sigma$ we get

$$
\begin{aligned}
D\left[p\left(\bar{x}_{3}\right), p\left(\bar{y}_{3}\right)\right]= & \sum_{\pi \in S_{3}} \sum_{\sigma \in S_{3}} D\left[x_{\pi(1)} x_{\pi(2)}, y_{\sigma(1)} y_{\sigma(2)}\right] x_{\pi(3)} y_{\sigma(3)} \\
& +\sum_{\pi \in S_{3}} \sum_{\sigma \in S_{3}} D\left(x_{\pi(1)} x_{\pi(2)}\right)\left[x_{\pi(3)}, y_{\sigma(1)} y_{\sigma(2)}\right] y_{\sigma(3)} \\
& +\sum_{\pi \in S_{3}} \sum_{\sigma \in S_{3}}\left[x_{\pi(1)} x_{\pi(2)}, y_{\sigma(1)} y_{\sigma(2)}\right] D\left(x_{\pi(3)}\right) y_{\sigma(3)} \\
& +\sum_{\pi \in S_{3}} \sum_{\sigma \in S_{3}} x_{\pi(1)} x_{\pi(2)} D\left[x_{\pi(3)}, y_{\sigma(1)} y_{\sigma(2)}\right] y_{\sigma(3)} \\
& +\sum_{\pi \in S_{3}} \sum_{\sigma \in S_{3}} D\left(y_{\sigma(1)} y_{\sigma(2)}\right)\left[x_{\pi(1)} x_{\pi(2)} x_{\pi(3)}, y_{\sigma(3)}\right] \\
& \left.+\sum_{\pi \in S_{3}} \sum_{\sigma \in S_{3}}\left[x_{\pi(1)} x_{\pi(2)} x_{\pi(3)}, y_{\sigma(1)} y_{\sigma(2)}\right)\right] D\left(y_{\sigma(3)}\right) \\
& +\sum_{\pi \in S_{3}} \sum_{\sigma \in S_{3}} y_{\sigma(1)} y_{\sigma(2)} D\left[x_{\pi(1)} x_{\pi(2)}, y_{\sigma(3)}\right] x_{\pi(3)} \\
& +\sum_{\pi \in S_{3}} \sum_{\sigma \in S_{3}} y_{\sigma(1)} y_{\sigma(2)} D\left(x_{\pi(1)} x_{\pi(2)}\right)\left[x_{\pi(3)}, y_{\sigma(3)}\right] \\
& +\sum_{\pi \in S_{3}} \sum_{\sigma \in S_{3}} y_{\sigma(1)} y_{\sigma(2)}\left[x_{\pi(1)} x_{\pi(2)}, y_{\sigma(3)}\right] D\left(x_{\pi(3)}\right) \\
& +\sum_{\pi \in S_{3}} \sum_{\sigma \in S_{3}} y_{\sigma(1)} y_{\sigma(2)} x_{\pi(1)} x_{\pi(2)} D\left[x_{\pi(3)}, y_{\sigma(3)}\right]
\end{aligned}
$$

It follows from both (10) and (11) that

(12)

$$
\begin{aligned}
0= & \sum_{\pi \in S_{3}} \sum_{\sigma \in S_{3}}\left(D\left[x_{\pi(1)} x_{\pi(2)}, y_{\sigma(1)} y_{\sigma(2)}\right]\right. \\
& +D\left(y_{\sigma(1)} y_{\sigma(2)}\right) x_{\pi(1)} x_{\pi(2)}-D\left(x_{\pi(1)} x_{\pi(2)}\right) y_{\sigma(1)} y_{\sigma(2)} \\
& \left.-x_{\pi(1)} x_{\pi(2)} D\left(y_{\sigma(1)} y_{\sigma(2)}\right)+y_{\sigma(1)} y_{\sigma(2)} D\left(x_{\pi(1)} x_{\pi(2)}\right)\right) y_{\sigma(3)} x_{\pi(3)} \\
& +\sum_{\pi \in S_{3}} \sum_{\sigma \in S_{3}}\left(x_{\pi(1)} x_{\pi(2)} D\left(y_{\sigma(1)} y_{\sigma(2)}\right)-D\left[x_{\pi(1)} x_{\pi(2)}, y_{\sigma(1)} y_{\sigma(2)}\right]\right. \\
& +D\left(x_{\pi(1)} x_{\pi(2)}\right) y_{\sigma(1)} y_{\sigma(2)}-D\left(y_{\sigma(1)} y_{\sigma(2)}\right) x_{\pi(1)} x_{\pi(2)} \\
& \left.-y_{\sigma(1)} y_{\sigma(2)} D\left(x_{\pi(1)} x_{\pi(2)}\right)\right) x_{\pi(3)} y_{\sigma(3)} \\
& +\sum_{\pi \in S_{3}} \sum_{\sigma \in S_{3}} x_{\pi(1)} x_{\pi(2)}\left(y_{\sigma(1)} y_{\sigma(2)} D\left(y_{\sigma(3)}\right) x_{\pi(3)}-y_{\sigma(1)} y_{\sigma(2)} x_{\pi(3)} D\left(y_{\sigma(3)}\right)\right. \\
& +y_{\sigma(1)} y_{\sigma(2)} D\left[x_{\pi(3)}, y_{\sigma(3)}\right]+y_{\sigma(1)} y_{\sigma(2)} y_{\sigma(3)} D\left(x_{\pi(3)}\right) \\
& \left.-y_{\sigma(1)} y_{\sigma(2)} D\left(x_{\pi(3)}\right) y_{\sigma(3)}\right)
\end{aligned}
$$




$$
\begin{aligned}
& +\sum_{\pi \in S_{3}} \sum_{\sigma \in S_{3}} y_{\sigma(1)} y_{\sigma(2)}\left(x_{\pi(1)} x_{\pi(2)} D\left(x_{\pi(3)}\right) y_{\sigma(3)}-x_{\pi(1)} x_{\pi(2)} D\left(y_{\sigma(3)}\right) x_{\pi(3)}\right. \\
& -x_{\pi(1)} x_{\pi(2)} y_{\sigma(3)} D\left(x_{\pi(3)}\right)-x_{\pi(1)} x_{\pi(2)} D\left[x_{\pi(3)}, y_{\sigma(3)}\right] \\
& \left.+x_{\pi(1)} x_{\pi(2)} x_{\pi(3)} D\left(y_{\sigma(3)}\right)\right)
\end{aligned}
$$

for all $x_{1}, x_{2}, x_{3}, y_{1}, y_{2}, y_{3} \in \mathcal{L}$. Define mappings $E, F: \mathcal{L}^{4} \rightarrow R$ by

$$
\begin{aligned}
E\left(u_{1}, u_{2}, u_{3}, u_{4}\right)= & D\left[u_{1} u_{2}, u_{3} u_{4}\right]+D\left(u_{3} u_{4}\right) u_{1} u_{2}-D\left(u_{1} u_{2}\right) u_{3} u_{4} \\
& -u_{1} u_{2} D\left(u_{3} u_{4}\right)+u_{3} u_{4} D\left(u_{1} u_{2}\right)
\end{aligned}
$$

and

$$
\begin{aligned}
F\left(u_{1}, u_{2}, u_{3}, u_{4}\right)= & u_{1} u_{2} D\left(u_{3}\right) u_{4}-u_{1} u_{2} D\left(u_{4}\right) u_{3}-u_{1} u_{2} u_{4} D\left(u_{3}\right) \\
& -u_{1} u_{2} D\left[u_{3}, u_{4}\right]+u_{1} u_{2} u_{3} D\left(u_{4}\right)
\end{aligned}
$$

for all $\bar{u}_{4} \in \mathcal{L}^{4}$. Accordingly, (12) can be rewritten as

$$
\begin{aligned}
0= & \sum_{\pi \in S_{3}} \sum_{\sigma \in S_{3}} E\left(x_{\pi(1)}, x_{\pi(2)}, y_{\sigma(1)}, y_{\sigma(2)}\right) y_{\sigma(3)} x_{\pi(3)} \\
& +\sum_{\pi \in S_{3}} \sum_{\sigma \in S_{3}} E\left(x_{\pi(1)}, x_{\pi(2)}, y_{\sigma(1)}, y_{\sigma(2)}\right) x_{\pi(3)} y_{\sigma(3)} \\
& +\sum_{\pi \in S_{3}} \sum_{\sigma \in S_{3}} x_{\pi(1)} x_{\pi(2)} F\left(x_{\pi(3)}, y_{\sigma(1)}, y_{\sigma(2)}, y_{\sigma(3)}\right) \\
& +\sum_{\pi \in S_{3}} \sum_{\sigma \in S_{3}} y_{\sigma(1)} y_{\sigma(2)} F\left(x_{\pi(1)}, x_{\pi(2)}, x_{\pi(3)}, y_{\sigma(3)}\right)
\end{aligned}
$$

for all $x_{1}, x_{2}, x_{3}, y_{1}, y_{2}, y_{3} \in \mathcal{L}$. Further, let $s: \mathbb{Z} \rightarrow \mathbb{Z}$ be a mapping defined by $s(i)=i-3$. For each $\sigma \in S_{3}$ the mapping $s^{-1} \sigma s:\{4,5,6\} \rightarrow\{4,5,6\}$ will be denoted by $\bar{\sigma}$. Then the last identity can be rewritten as

$$
\begin{aligned}
0= & \sum_{\pi \in S_{3}} \sum_{\sigma \in S_{3}} E\left(x_{\pi(1)}, x_{\pi(2)}, x_{\bar{\sigma}(4)}, x_{\bar{\sigma}(5)}\right) x_{\bar{\sigma}(6)} x_{\pi(3)} \\
& +\sum_{\pi \in S_{3}} \sum_{\sigma \in S_{3}} E\left(x_{\pi(1)}, x_{\pi(2)}, x_{\bar{\sigma}(4)}, x_{\bar{\sigma}(5)}\right) x_{\pi(3)} x_{\bar{\sigma}(6)} \\
& +\sum_{\pi \in S_{3}} \sum_{\sigma \in S_{3}} x_{\pi(1)} x_{\pi(2)} F\left(x_{\pi(3)}, x_{\bar{\sigma}(4)}, x_{\bar{\sigma}(5)}, x_{\bar{\sigma}(6)}\right) \\
& +\sum_{\pi \in S_{3}} \sum_{\sigma \in S_{3}} x_{\bar{\sigma}(4)} x_{\bar{\sigma}(5)} F\left(x_{\pi(1)}, x_{\pi(2)}, x_{\pi(3)}, x_{\bar{\sigma}(6)}\right)
\end{aligned}
$$

for all $x_{1}, \ldots, x_{6} \in \mathcal{L}$. Now we simply apply the definition of 6 -freeness $\mathcal{L}$. There exist mappings $p_{6, j}: \mathcal{L}^{4} \rightarrow R, j=1, \ldots, 5$, and $\lambda_{6}: \mathcal{L}^{5} \rightarrow C(\mathcal{L})$ such that

(13) $\sum_{\substack{\pi \in S_{3} \\ \pi(1)=1}} \sum_{\sigma \in S_{3}} x_{\pi(2)} F\left(x_{\pi(3)}, x_{\bar{\sigma}(4)}, x_{\bar{\sigma}(5)}, x_{\bar{\sigma}(6)}\right)=\sum_{j=1}^{5} p_{6, j}\left(\bar{x}_{5}^{j}\right) x_{j}+\lambda_{6}\left(\bar{x}_{5}\right)$ 
for all $\bar{x}_{5} \in \mathcal{L}^{5}$. Note that $(13)$ can be rewritten as

(14) $\sum_{i=1}^{5} x_{i}\left(\sum_{\substack{\pi \in S_{3} \\ \pi(1)=1 \\ \pi(2)=i}} \sum_{\sigma \in S_{3}} F\left(x_{\pi(3)}, x_{\bar{\sigma}(4)}, x_{\bar{\sigma}(5)}, x_{\bar{\sigma}(6)}\right)\right)-\sum_{j=1}^{5} p_{6, j}\left(\bar{x}_{5}^{j}\right) x_{j} \in C(\mathcal{L})$

for all $\bar{x}_{5} \in \mathcal{L}^{5}$. Recalling the definition of a mapping $F$ and using (14) we get

$$
\begin{aligned}
& \sum_{\substack{\pi \in S_{3} \\
\text { a(1)=1 } \\
\pi(2)=2}} \sum_{\sigma \in S_{3}}\left(x_{\bar{\sigma}(4)} x_{\bar{\sigma}(5)} D\left(x_{\bar{\sigma}(6)}\right) x_{\pi(3)}-x_{\bar{\sigma}(4)} x_{\bar{\sigma}(5)} x_{\pi(3)} D\left(x_{\bar{\sigma}(6)}\right)\right. \\
& -x_{\bar{\sigma}(4)} x_{\bar{\sigma}(5)} D\left[x_{\pi(3)}, x_{\bar{\sigma}(6)}\right]+x_{\bar{\sigma}(4)} x_{\bar{\sigma}(5)} x_{\bar{\sigma}(6)} D\left(x_{\pi(3)}\right) \\
& \left.-x_{\bar{\sigma}(4)} x_{\bar{\sigma}(5)} D\left(x_{\pi(3)}\right) x_{\bar{\sigma}(6)}\right)-\sum_{j=1}^{4} p_{5, j}\left(\bar{x}_{4}^{j}\right) x_{j} \in C(\mathcal{L})
\end{aligned}
$$

for all $\bar{x}_{4} \in \mathcal{L}^{4}$ and some mappings $p_{5, j}: \mathcal{L}^{3} \rightarrow R$. Since $\mathcal{L}$ is 6 -free, after a finite number of steps we arrive at

$$
D(x) y-D(y) x-y D(x)+D[y, x]+x D(y)=f(x) y+g(y) x+\mu(x, y)
$$

for all $x, y \in \mathcal{L}$, where $f, g: \mathcal{L} \rightarrow R$ and $\mu: \mathcal{L}^{2} \rightarrow C(\mathcal{L})$. Hence

(15) $D[x, y]=D(x) y-D(y) x-y D(x)+x D(y)-f(x) y-g(y) x-\mu(x, y)$.

If we replace the roles of denotations $x$ and $y$ in (15) and compare so obtained identities we arrive at $0=-f(x) y-g(y) x-\mu(x, y)-f(y) x-g(x) y-\mu(y, x)$. Since $\mathcal{L}$ is a 6 -free subset of $R$ we have $-f(x)-g(x)=0$ for all $x \in \mathcal{L}$. We also obtain $\mu(x, x)=0$ and $\mu(x, y)+\mu(y, x)=0$ for all $x, y \in \mathcal{L}$. From (15) it follows that $D\left[x^{2}, x\right]=0$. Hence

$$
\begin{aligned}
0= & D\left(x^{2}\right) x-D(x) x^{2}-x D\left(x^{2}\right)+x^{2} D(x) \\
& -f\left(x^{2}\right) x+f(x) x^{2}-\mu\left(x^{2}, x\right)
\end{aligned}
$$

for all $x \in \mathcal{L}$. After a complete linearization of this identity we arrive at

$$
-D(x y)-D(y x)+x D(y)+y D(x)=h(x) y+k(y) x+\mu^{\prime}(x, y)
$$

and also

$$
-D(y x)-D(x y)+y D(x)+x D(y)=h(y) x+k(x) y+\mu^{\prime}(y, x)
$$

for all $x, y \in \mathcal{L}$, where $h, k: \mathcal{L} \rightarrow R$ and $\mu^{\prime}: \mathcal{L}^{2} \rightarrow C(\mathcal{L})$. From the last two identities we obtain $0=h(x) y+k(y) x-h(y) x-k(x) y+\mu^{\prime}(x, y)-\mu^{\prime}(y, x)$, which yields $h(x)=k(x), \mu^{\prime}(x, y)=\mu^{\prime}(y, x)$ for all $x, y \in \mathcal{L}$. If $y=x$ in $(17)$ we have

$$
2 D\left(x^{2}\right)=2 x D(x)-2 h(x) x-\mu^{\prime}(x, x) .
$$

Using this identity in (16) we obtain

(20) $f\left(x^{2}\right) x=x D(x) x-h(x) x^{2}-D(x) x^{2}+f(x) x^{2}+x h(x) x-\mu\left(x^{2}, x\right)$. 
Replacing $y$ for $x$ and $x$ for $x^{2}$ in (17) and using (5) and (19) we arrive at

$$
2 h\left(x^{2}\right) x=2 h(x) x^{2}-2 x h(x) x-4 x D(x) x-2 \mu^{\prime}\left(x^{2}, x\right)+\mu^{\prime}(x, x) x .
$$

From (17) we get $D(y x)=-D(x y)+x D(y)+y D(x)-h(x) y-h(y) x-\mu^{\prime}(x, y)$. Using this relation in (15) we get

$$
\begin{aligned}
2 D(x y)= & D(x) y-D(y) x+2 x D(y)-f(x) y+f(y) x \\
& -h(x) y-h(y) x-\mu(x, y)-\mu^{\prime}(x, y) .
\end{aligned}
$$

From (22) we get

$$
\begin{aligned}
2 D\left(x^{4}\right)= & D(x) x^{3}-D\left(x^{3}\right) x+2 x D\left(x^{3}\right)-f(x) x^{3}+f\left(x^{3}\right) x \\
& -h(x) x^{3}-h\left(x^{3}\right) x-\mu\left(x, x^{3}\right)-\mu^{\prime}\left(x, x^{3}\right)
\end{aligned}
$$

and also

$$
\begin{aligned}
2 D\left(x^{4}\right)= & D\left(x^{3}\right) x-D(x) x^{3}+2 x^{3} D(x)-f\left(x^{3}\right) x+f(x) x^{3} \\
& -h\left(x^{3}\right) x-h(x) x^{3}-\mu\left(x^{3}, x\right)-\mu^{\prime}\left(x^{3}, x\right) .
\end{aligned}
$$

Comparing these two identities, using (5), (19) and (20) we obtain

$$
f\left(x^{3}\right) x=f\left(x^{2}\right) x^{2}+\mu\left(x^{2}, x\right) x-\mu\left(x^{3}, x\right) .
$$

From (19) we arrive at $2 D\left(x^{4}\right)=2 x^{2} D\left(x^{2}\right)-2 h\left(x^{2}\right) x^{2}-\mu^{\prime}\left(x^{2}, x^{2}\right)$. Comparing this relation with (23), using (5), (19), (20), (21) and (24) we get

$$
\begin{aligned}
2 h\left(x^{3}\right) x= & 2 x^{2} D(x) x+2 h(x) x^{3}-6 x h(x) x^{2}+4 x^{2} h(x) x-8 x D(x) x^{2} \\
& -2 \mu^{\prime}\left(x^{3}, x\right)+3 \mu^{\prime}(x, x) x^{2}-4 \mu^{\prime}\left(x^{2}, x\right) x+2 \mu^{\prime}\left(x^{2}, x^{2}\right) .
\end{aligned}
$$

From (5) we get $2 D\left(x^{6}\right)=2 D\left(x^{4}\right) x^{2}+2 x^{4} D\left(x^{2}\right)$. On the other hand from (19) we obtain $2 D\left(x^{6}\right)=2 x^{3} D\left(x^{3}\right)-2 h\left(x^{3}\right) x^{3}-\mu^{\prime}\left(x^{3}, x^{3}\right)$. Comparing these two identities and using (5), (19), (21) and (25) we get

$$
\begin{aligned}
0= & -2 x^{2} h(x) x^{3}+4 x D(x) x^{4}+4 x h(x) x^{4}-2 x^{2} D(x) x^{3}+2 x^{4} D(x) x \\
& -2 x^{3} h(x) x^{2}-2 x^{3} D(x) x^{2}+2 x^{4} h(x) x-\mu^{\prime}(x, x) x^{4} \\
& -\mu^{\prime}\left(x^{2}, x^{2}\right) x^{2}+2 \mu^{\prime}\left(x^{3}, x\right) x^{2}+2 \mu^{\prime}\left(x^{2}, x\right) x^{3}-\mu^{\prime}\left(x^{3}, x^{3}\right) .
\end{aligned}
$$

After a complete linearization of the last identity, we use the 6 -freeness of $\mathcal{L}$ and then replace all $x_{i}$ for $x$, where $i=1, \ldots, 6$. Therefore

$$
\begin{aligned}
0= & -2 x h(x) x^{2}+4 D(x) x^{3}+4 h(x) x^{3}-2 x D(x) x^{2} \\
& +2 x^{3} D(x)-2 x^{2} h(x) x-2 x^{2} D(x) x+2 x^{3} h(x) \\
& +2 \mu^{\prime}\left(x^{3}, x\right)+2 \mu^{\prime}\left(x^{2}, x\right) x-\mu^{\prime}(x, x) x^{2}-\mu^{\prime}\left(x^{2}, x^{2}\right) .
\end{aligned}
$$

Since $\mathcal{L}$ is a 6 -free subset of $R$, it is easy to verify that $4 h(x)+4 D(x)=$ $x p+\lambda(x)$, for all $x \in \mathcal{L}$, where $p \in R$ and $\lambda: \mathcal{L} \rightarrow C(\mathcal{L})$. Using $4 h(x)=$ $-4 D(x)+x p+\lambda(x)$ in (19) we get

$$
4 D\left(x^{2}\right)=4 x D(x)+4 D(x) x-x p x-\lambda(x) x-2 \mu^{\prime}(x, x) .
$$


From (21) we obtain

$$
\begin{aligned}
-4 D\left(x^{2}\right) & x+x^{2} p x+\lambda\left(x^{2}\right) x \\
= & -4 D(x) x^{2}+x p x^{2}+\lambda(x) x^{2}+4 x D(x) x \\
& -x^{2} p x-\lambda(x) x^{2}-8 x D(x) x-4 \mu^{\prime}\left(x^{2}, x\right)+2 \mu^{\prime}(x, x) x .
\end{aligned}
$$

Using (27) in the last identity we obtain $-2 x^{2} p x=x^{2} \lambda(x)+x \lambda\left(x^{2}\right)+$ $4 \mu^{\prime}\left(x^{2}, x\right)$. Since $\mathcal{L}$ is a 6 -free subset of $R$ we get $-2 p x=\lambda(x)$ and then $-2 p x \in C(\mathcal{L})$ for all $x \in R$. Thus $p z[x, y]=0$ for all $x, y, z \in R$. Since $R$ is a prime ring it follows that $p=0$ or $R$ is commutative. If $p=0$, we get $\lambda=0$ and then $4 h(x)+4 D(x)=0$. If $R$ is commutative we can prove the same. Consequently from (20) we obtain $f\left(x^{2}\right) x=f(x) x^{2}-\mu\left(x^{2}, x\right)$ and then from (16) also $D\left(x^{2}\right) x+x^{2} D(x)=D(x) x^{2}+x D\left(x^{2}\right)$. We use this identity and $h(x)=-D(x)$ in $(17)$ and obtain $2 D\left(x^{3}\right)=2 D\left(x^{2}\right) x+2 x^{2} D(x)-\mu^{\prime}\left(x^{2}, x\right)$. On the other hand from (5) we have $2 D\left(x^{3}\right)=2 D\left(x^{2}\right) x+2 x^{2} D(x)$. It follows that $\mu^{\prime}\left(x^{2}, x\right)=0$. We use this identity and $h(x)=-D(x)$ in (26), which implies $-\mu^{\prime}(x, x) x^{2}-\mu^{\prime}\left(x^{2}, x^{2}\right)+2 \mu^{\prime}\left(x^{3}, x\right)=0$. Thus $\sum_{\pi \in S_{4}} \mu^{\prime}\left(x_{\pi(1)}, x_{\pi(2)}\right) x_{\pi(3)} x_{\pi_{(4)}} \in C(\mathcal{L})$. We use the 6 -freeness of $\mathcal{L}$ and obtain $\mu^{\prime}(x, y)=0$ for all $x, y \in \mathcal{L}$. Consequently from (19) it follows that $D$ is a Jordan derivation. By Herstein's theorem ([11]) $D$ is a derivation. Thereby the proof is completed.

We are now in the position to prove Theorem 3 .

Proof of Theorem 3. The complete linearization of (5) gives us (8). Assume first that $R$ is not a PI ring. According to Theorem $4 D$ is a derivation.

Now suppose that $R$ is a PI ring. It is well-known that in this case $R$ has a nonzero center (see [12]). Let $c$ be a nonzero central element. Pick any $x \in R$ and set $x_{1}=x_{2}=c x$ and $x_{3}=x$ in (8). Hence we obtain

$$
3 D\left(c^{2} x^{3}\right)=D\left(c^{2} x^{2}\right) x+2 D\left(c x^{2}\right) c x+c^{2} x^{2} D(x)+2 c x^{2} D(c x) .
$$

Next, setting $x_{1}=x_{2}=c$ and $x_{3}=x^{3}$ in (8) we arrive at

$$
\begin{aligned}
3 D\left(c^{2} x^{3}\right) & =D\left(c^{2}\right) x^{3}+2 D\left(c x^{3}\right) c+c^{2} D\left(x^{3}\right)+2 c x^{3} D(c) \\
& =D\left(c^{2}\right) x^{3}+2 D\left(c x^{3}\right) c+c^{2}\left(D\left(x^{2}\right) x+x^{2} D(x)\right)+2 c x^{3} D(c)
\end{aligned}
$$

for all $x \in R$. Comparing both identities we get

$$
\begin{aligned}
& D\left(c^{2} x^{2}\right) x+2 D\left(c x^{2}\right) c x+2 c x^{2} D(c x) \\
& \quad=D\left(c^{2}\right) x^{3}+2 D\left(c x^{3}\right) c+c^{2} D\left(x^{2}\right) x+2 c x^{3} D(c)
\end{aligned}
$$

for all $x \in R$. Setting $x_{1}=x$ and $x_{2}=x_{3}=c$ in the complete linearization of (28) we get

$$
c^{2} D(c) x+2 D\left(c^{2} x\right) c+2 c x D\left(c^{2}\right)=2 D\left(c^{3} x\right)+3 x c^{2} D(c)
$$


for all $x \in R$. Then substituting $x$ for $c x$ in relation (29) we obtain

$$
D(c) c^{3} x+2 D\left(c^{3} x\right) c+2 c^{2} x D\left(c^{2}\right)=2 D\left(c^{4} x\right)+3 c^{3} x D(c) .
$$

Multiplying identity (29) by $c$ we get

$$
D(c) c^{3} x+2 D\left(c^{2} x\right) c^{2}+2 c^{2} x D\left(c^{2}\right)=2 D\left(c^{3} x\right) c+3 c^{3} x D(c) .
$$

Comparing the last two identities, we have

$$
2 D\left(c^{3} x\right) c=D\left(c^{4} x\right)+D\left(c^{2} x\right) c^{2} .
$$

for all $x \in R$. Substituting $x$ by $c x$ in (5) we get

$$
3 D\left(c^{3} x^{3}\right)=3 D\left(c^{2} x^{2}\right) c x+3 c^{2} x^{2} D(c x)
$$

for all $x \in R$. Next, setting $x_{1}=x_{2}=c$ and $x_{3}=c x^{3}$ in the complete linearization of (5) we have

$$
3 D\left(c^{3} x^{3}\right)=D\left(c^{2}\right) c x^{3}+2 c D\left(c^{2} x^{3}\right)+c^{2} D\left(c x^{3}\right)+2 c^{2} x^{3} D(c) .
$$

Comparing the last two identities we see that

(32) $3 D\left(c^{2} x^{2}\right) x+3 c x^{2} D(c x)=D\left(c^{2}\right) x^{3}+2 D\left(c^{2} x^{3}\right)+c D\left(c x^{3}\right)+2 c x^{3} D(c)$.

Setting $x_{1}=x_{2}=c$ and $x_{3}=x$ in the complete linearization of (32) and using (31) we get

$$
D\left(c^{2}\right) c x+D(c x) c^{2}+2 x D\left(c^{2}\right) c+2 D\left(c^{2} x\right) c=3 D\left(c^{3} x\right)+2 x D(c) c^{2} .
$$

Using the last identity and (30) we obtain

$$
D\left(c^{2}\right) c x+D(c x) c^{2}+x D(c) c^{2}=D\left(c^{3} x\right)+D(c) x c^{2}
$$

and so

$$
D\left(c^{3} x\right)=D(c x) c^{2}+D\left(c^{2}\right) c x+[x, D(c)] c^{2}
$$

for all $x \in R$. Setting $x_{1}=x_{2}=c$ and $x_{3}=c x$ in (8) we arrive at

$$
6 D\left(c^{3} x\right)=2 D\left(c^{2}\right) c x+4 D\left(c^{2} x\right) c+2 c^{2} D(c x)+4 c^{2} x D(c) .
$$

Comparing the last two identities we obtain

$$
2 D(c x) c+2 D\left(c^{2}\right) c x+3[x, D(c)] c=2 D\left(c^{2} x\right)+2 c x D(c)
$$

and use (33) in (34) we get

$$
2 D\left(c^{2} x\right)-2 D(c x) c=D(c) x c+x c D(c) .
$$

Substituting $x$ for $c x$ in (33), using (31) and (34) we have

$$
D\left(c^{2}\right) c^{2} x=2 D(c) c^{3} x
$$

for all $x \in R$. If $x=c$, we get $D\left(c^{2}\right)=2 D(c) c$ and then from (35) also $D\left(c^{3}\right)=3 D(c) c^{2}$. Next we set $x_{1}=x_{2}=c$ and $x_{3}=x$ in (8). This yields

$$
6 D\left(c^{2} x\right)=2 D\left(c^{2}\right) x+4 D(c x) c+2 c^{2} D(x)+4 c x D(c) .
$$

Using (35) we obtain

$$
2 D(c x)-2 D(x) c=D(c) x+x D(c)
$$


and so

$$
2 D\left(c x^{2}\right)-2 D\left(x^{2}\right) c=D(c) x^{2}+x^{2} D(c)
$$

for all $x \in R$. Setting $x_{1}=x_{2}=x$ and $x_{3}=c$ in (8), using (37) and (38) we have

$$
\begin{aligned}
6 D\left(c x^{2}\right)= & 2 D\left(x^{2}\right) c+4 D(c x) x+2 x^{2} D(c)+4 c x D(x) \\
= & \left(2 D\left(c x^{2}\right)-D(c) x^{2}-x^{2} D(c)\right) \\
& +4 D(c x) x+2 x(x D(c)+2 c D(x)) \\
= & 2 D\left(c x^{2}\right)-D(c) x^{2}-x^{2} D(c) \\
& +4 D(c x) x+4 x D(c x)-2 x D(c) x .
\end{aligned}
$$

Comparing this identity and (38) we get

$$
4 D(c x) x+4 x D(c x)-2 x D(c) x=3 D(c) x^{2}+3 x^{2} D(c)+4 D\left(x^{2}\right) c
$$

and so

(40) $4 D\left(x^{2}\right) c=4(D(c x) x+x D(c x))-3\left(D(c) x^{2}+x^{2} D(c)\right)-2 x D(c) x$.

Then we use (38) in this relation and we obtain

$$
3 D\left(c x^{2}\right)-D\left(x^{2}\right) c=2 D(c x) x+2 x D(c x)-x D(c) x .
$$

But on the other hand we use (37), (38) in (39) we have

$$
2 D\left(c x^{2}\right)+2 D\left(x^{2}\right) c=4 D(x) x c+4 x D(x) c+2 x D(c) x .
$$

Again comparing the last two identities we obtain

$$
2 D\left(c x^{2}\right)=D(c x) x+x D(c x)+D(x) x c+x c D(x)
$$

for all $x \in R$. From the first line of identity (39) we get

$$
D\left(x^{2}\right) c=3 D\left(c x^{2}\right)-2 D(c x) x-x^{2} D(c)-2 c x D(x) .
$$

Then we use (37), (41) in (42) and obtain

$$
\begin{aligned}
2 D\left(x^{2}\right) c= & 3 D(c x) x+3 x D(c x)+3 D(x) x c+3 x D(x) c \\
& -4 D(c x) x-2 x(x D(c)+2 c D(x)) \\
= & 3 x D(c x)+3 D(x) x c+3 x D(x) c \\
& -D(c x) x-2 x(2 D(c x)-D(c) x) \\
= & 3(D(x) x+x D(x)) c-(D(c x) x+x D(c x))+2 x D(c) x .
\end{aligned}
$$

Using the last two identities in (38) we arrive at

(43) $D(c) x^{2}+x^{2} D(c)=2(D(c x) x+x D(c x))-2(D(x) x c+x c D(x))-2 x D(c) x$.

Then we use (37) in (40) and we get

$$
4 D\left(x^{2}\right) c=4(D(x) x c+c x D(x))-\left(D(c) x^{2}+x^{2} D(c)\right)+2 x D(c) x
$$


and also

$$
4 D\left(x^{2}\right) c^{2}=4\left(D(x) x c^{2}+c^{2} x D(x)\right)-\left(D\left(c^{2}\right) x^{2}+x^{2} D\left(c^{2}\right)\right)+2 x D\left(c^{2}\right) x .
$$

Multiplying identity (44) by $c$ we have

$$
4 D\left(x^{2}\right) c^{2}=4\left(D(x) x c^{2}+c^{2} x D(x)\right)-\left(D(c) x^{2} c+c x^{2} D(c)\right)+2 x D(c) c x .
$$

Comparing the last two identities we have

$$
D(c) x^{2}+x^{2} D(c)=2 x D(c) x .
$$

Using this in (43) we get

$$
D(c x) x+x D(c x)=D(x) x c+c x D(x)+2 x D(c) x .
$$

Now we use the last identity and (45) in (40) and we obtain $D\left(x^{2}\right)=D(x) x+$ $x D(x)$ for all $x \in R$. In other words, $D$ is a Jordan derivation. By Herstein's theorem $D$ is a derivation. The proof of the theorem is complete. The proof in case we have the relation (6) goes through in a similar way and will be omitted.

\section{REFERENCES}

[1] K. I. Beidar, M. Brešar, M. A. Chebotar and W. S. Martindale 3rd, On Herstein's Lie map conjectures. II, J. Algebra 238 (2001), 239-264.

[2] K. I. Beidar and Y. Fong, On additive isomorphisms of prime rings preserving polynomials, J. Algebra 217 (1999), 650-667.

[3] M. Brešar, Jordan derivations on semiprime rings, Proc. Amer. Math. Soc. 104 (1988), 1003-1006.

[4] M. Brešar, Jordan mappings of semiprime rings, J. Algebra 127 (1989), 218-228.

[5] M. Brešar, Functional identities: a survey, Contemp. Math. 259 (2000), 93-109.

[6] M. Brešar, M. A. Chebotar and W. S. Martindale 3rd, Functional identities, Birkhäuser Verlag, Basel, 2007.

[7] M. Brešar and J. Vukman, Jordan derivations on prime rings, Bull. Austral. Math. Soc. 37 (1988), 321-323.

[8] M. Brešar and J. Vukman, Jordan $(\theta, \phi)$-derivations, Glas. Mat. Ser. III 26(46) (1991), $13-17$.

[9] Cheng-Kai Liu and Wen-Kwei Shiue, Generalized Jordan triple $(\theta, \phi)$-derivations on semiprime rings, Taiwanese J. Math. 11 (2007), 1397-1406.

[10] J. M. Cusack, Jordan derivations on rings, Proc. Amer. Math. Soc. 53 (1975), 321-324.

[11] I. N. Herstein, Jordan derivations of prime rings, Proc. Amer. Math. Soc. 8 (1957), 1104-1110.

[12] L. Rowen, Some results on the center of a ring with polynomial identity, Bull. Amer. Math. Soc. 79 (1973), 219-223.

[13] J. Vukman, On derivations of standard operator algebras and semisimple $H^{*}$-algebras, Studia Sci. Math. Hungar. 44 (2007), 57-63.

[14] J. Vukman, Some remarks on derivations in semiprime rings and standard operator algebras, Glas. Mat. Ser. III 46(66) (2011), 43-48.

[15] J. Vukman, I. Kosi-Ulbl and D. Eremita, On certain equations in rings, Bull. Austral. Math. Soc. 71 (2005), 53-60. 
M. Fošner

Faculty of logistics

University of Maribor

Mariborska cesta 7, 3000 Celje

Slovenia

E-mail: maja.fosner@uni-mb.si

N. Peršin

Prušnikova 48, 2000 Maribor

Slovenia

E-mail: nina_persin@t-2.net

Received: 22.1.2012. 